\title{
A robust test for assortative mating
}

\author{
Emmanuelle Génin ${ }^{1}$, Carole Ober ${ }^{2}$, Lowell Weitkamp ${ }^{3}$ and Glenys Thomson ${ }^{1}$
}

\author{
${ }^{1}$ D epartment of Integrative Biology, University of California, Berkeley, CA, USA; ${ }^{2}$ Department of Human Genetics, \\ University of Chicago, Chicago, IL, USA; ${ }^{3}$ Department of Psychiatry and Division of Genetics, University of \\ Rochester Medical Center, Rochester, NY, USA
}

Testing for random mating in human populations is difficult due to confounding factors such as ethnic preference and population stratification. With HLA, the high level of polymorphism is an additional problem since it is rare for couples to share the same haplotype. Focus on an ethnically homogeneous population, where levels of polymorphism at HLA loci are more limited, may provide the best situation in which to detect non-random mating. However, such populations are often genetic isolates where there may be inbreeding to an extent that is difficult to quantify and account for. We have developed a test for random mating at a multiallelic locus that is robust to stratification and inbreeding. This test relies on the availability of genotypic information from the parents of both spouses. The focus of the test is on families where there is allele sharing between the parents of both spouses, so that potential spouses could share an allele. Denoting the shared allele at the locus of interest by $A$, then under the assumption of random mating, heterozygous parents $A X$ should transmit allele $A$ equally as frequently as allele $X$ to their offspring. When there is positive (negative) assortative mating, A will be transmitted more (less) often than $X$. The power of the test has been computed in a number of situations. Data on high resolution HLA haplotypes from the Hutterite population were reinvestigated by the proposed test. The test detects significant negative assortative mating when the parental origin of the shared haplotype is taken into account. European Journal of Human Genetics (2000) 8, 119-124.

Keywords: assortative mating; HLA; Hutterites; power; robustness; inbreeding; admixture

\section{Introduction}

Mate choice in animal and human populations has been the subject of many investigations. Assortative mating has long been recognized in humans for characteristics such as stature and congenital deafness, ${ }^{1}$ which involve both genetic and environmental factors. Studies in mice have implicated genes from the major histocompatibility complex (MHC) in mate choice; female mice generally prefer mating with males different from themselves for genes in the $\mathrm{H} 2$ region. $^{2}$ However, attempts to test for an effect of HLA on mate choice in humans have led to equivocal conclusions. ${ }^{3-6}$

Confounding factors such as ethnic preference and population stratification make testing of random mating in human populations difficult. Another problem specific to human

Correspondence: Emmanuelle Génin, INSERM U535, Bâtiment INSERM Gregory Pincus, secteur marron, porte 15, 80 rue du Général Leclerc, 94276 Le Kremlin Bicêtre Cedex. Tel: + 33149595330 ;

Fax: + 33149595331 ; E-mail: genin@ccr.jussieu.fr

Received 7 June 1999; revised 7 September 1999; accepted 23

September 1999 leukocyte antigens (HLA) is the very high level of polymorphism in this region that lowers the expectation of finding spouses sharing an identical allele or haplotype. It has therefore been suggested that studies of mate choice in humans should focus on ethnically homogeneous populations, where levels of polymorphism at HLA loci is more limited. However, such populations are often genetic isolates in which there may be considerable inbreeding or, alternatively, avoidance of mating with close relatives to an extent that is difficult to quantify and account for.

The purpose of this study is to propose a test for assortative mating that is not sensitive to stratification and/or inbreeding. This test relies on the availability of genotypic information for parents of both spouses. The power of the test is computed for different models of positive and negative assortative mating and allowing for different types of relationships between the spouses. The properties of the test are compared to those of one commonly used test of assortative mating based on the distribution of the number of alleles shared by spouses. ${ }^{4-6}$ 
Recently, two studies in human isolates have been published, ${ }^{5,6}$ The first, in the Hutterite population, showed evidence of negative assortative mating for HLA: spouses matched less often for HLA haplotypes than expected. ${ }^{5}$ This effect was not found when each locus was considered individually, suggesting a haplotype effect. ${ }^{7}$ The second study, in South Amerindian tribes, showed no deviation from random mating when analyzing each locus separately. In both analyses, assortative mating was tested by comparing the observed distribution of alleles or haplotypes shared by spouses to the one expected under random mating and the significance was dependent on some hypotheses regarding the structure of the population. In this paper, we reanalyze the Hutterite data of Ober et $\mathrm{al}^{5}$ with the new test we have proposed, which does not depend on any hypotheses regarding the population structure.

\section{Material and methods \\ Description of the mating test}

The robust test we have developed relies on the availability of genotypic information for the parents (generation $\mathrm{G}_{0}$ ) of both spouses (generation $G_{1}$ ), and focuses only on families where the parents share an allele, called $A_{i}$, at the test locus under study (see Table1). The pair of parents sharing a common allele is called an informative pair. Under the assumption of random mating at generation $\mathrm{G}_{1}$, parents that are heterozygous $A_{i} A_{j}$ transmit al lele $A_{i}$ as often as allele $A_{j}$ to their offspring. If the shared allele $A_{i}$ is transmitted more often, it means that in generation $G_{1}$, there was positive assortative mating with respect to the locus under study or a locus in linkage disequilibrium with that locus (see discussion). Conversely, if $A_{i}$ is transmitted less often than $A_{j}$, it means that in generation $G_{1}$, there was negative assortative mating at that locus.

Let $N$ be the number of heterozygous parents $A_{i} A_{j}$ in informative pairs (we will refer to these parents as informative parents in the following). Let $\mathrm{T}$ be the number of cases where informative parents have transmitted the shared allele $A_{i}$ to their offspring (one of the spouses in generation $G_{1}$ ). The statistic $M$ defined by:

$$
M=\frac{4(T-N / 2)^{2}}{N}
$$

is distributed as a chi-square with one degree of freedom under $\mathrm{H}_{0}$. The test we propose is based on this statistic and will be called the $M$ test.

Depending on the structure of the family, the number of informative pairs and informative parents may vary (see Table1). Special attention is drawn to the case of families where one parent shares one allele in common with both parents of his child's in-laws, that is families in which the parents of the first spouse $\operatorname{are}_{i} A_{j}, A_{k} A_{l}$ and the parents of the second spouse are $A_{i} A_{r}, A_{j} A_{s}$. In these families, only the parents of the second spouse should be considered as informative with the shared allele being respectively $A_{i}$ and $A_{j}$. The consequence is that the test requires at least three alleles be present in the family. Therefore it cannot be used with biallelic markers. Indeed, with biallelic markers, heterozygous parents can only share alleles with both parents of their child's in-laws.

\section{Statistical properties of the M test compared with the IBS test}

The statistical properties of the M test (robustness and power) have been studied by simulations. A comparison is performed with the classically used identical by state (IBS) test. ${ }^{4-6}$

Description of the IBS test The IBS test is also a chi-square test that compares the observed distribution of spouses sharing 0,1 or 2 IBS alleles to the distribution expected under random mating $\left(\mathrm{P}_{0}, \mathrm{P}_{1}, \mathrm{P}_{2}\right)$. To avoid any assumption regarding the mating pattern in the parental generation, Hedrick ${ }^{8}$ proposed estimating $\mathrm{P}_{0}, \mathrm{P}_{1}$ and $\mathrm{P}_{2}$ from the data by

Table 1 Families considered in the analysis

\begin{tabular}{|c|c|c|c|c|c|}
\hline Type & Parents of 1st spouse & Parents of 2 nd spouse & & $\begin{array}{l}\text { No. of informative } \\
\text { pairs per family }\end{array}$ & $\begin{array}{l}\text { No. of informative } \\
\text { parents }\end{array}$ \\
\hline 1 & $A_{1} A_{j} A_{k} A_{1}$ & $A A_{m} A_{r} A_{s}$ & $\begin{array}{l}\mathrm{i} \neq \mathrm{j}, \mathrm{k}, \mathrm{I} \\
\mathrm{m}, \mathrm{r}, \mathrm{s} \neq \mathrm{i}, \mathrm{k}, \mathrm{I}\end{array}$ & 1 & 2 \\
\hline 2 & $A_{1} A_{i} A_{k}$ & $A_{1} A_{m} A_{r} A_{s}$ & $\mathrm{i} \neq \mathrm{k}, \mathrm{I}$ & 1 & 1 \\
\hline 3 & $A_{i} A_{j} A_{k}$ & $A_{i} A_{m} A_{k} A_{r}$ & $\begin{array}{l}\mathrm{m}, \mathrm{l}, \mathrm{S} \neq \mathrm{l}, \mathrm{K}, \mathrm{I} \\
\mathrm{i} \neq \mathrm{j}, \mathrm{k}, \mathrm{I} \\
\mathrm{l} \neq \mathrm{k}\end{array}$ & 2 & 4 \\
\hline 4 & $A, A_{i} A_{1}$ & $A A_{m} A_{k} A_{r}$ & $\begin{array}{l}m, r \neq i, j, k, l \\
i \neq k, I \\
l \neq k\end{array}$ & 2 & 3 \\
\hline 5 & $\mathrm{~A}_{i} \mathrm{~A}_{\mathrm{k}} \mathrm{A}_{\mathrm{k}}$ & $\mathrm{A}_{\mathrm{r}} \mathrm{A}_{\mathrm{m}} \mathrm{A}_{\mathrm{k}} \mathrm{A}_{\mathrm{r}}$ & $\begin{array}{l}m, r \neq i, k, l \\
i \neq k, m \\
m \neq k \\
r \neq i, j, k\end{array}$ & 2 & 2 \\
\hline 6 & $A A_{j} A_{k} A$ & $A_{i} A_{m}, A_{j}$ & $\begin{array}{l}\mathrm{i} \neq \mathrm{j}, \mathrm{k}, \mathrm{I} \\
\mathrm{m}, \mathrm{r} \neq \mathrm{i}, \mathrm{j}, \mathrm{k}, \mathrm{I}\end{array}$ & 2 & 2 \\
\hline
\end{tabular}


using the observed female and male genotypic frequencies in mating pairs from the population.

\section{Robustness of the IBS and M tests to stratification and} inbreeding We investigated the problem of population stratification that may arise if the population investigated is not homogeneous. For simplification, we have considered a population with two groups. We assumed that mating is completely endogamous and random in the generation of the parents (generation $\mathrm{G}_{0}$ ), and in the generation studied (generation $G_{1}$ ) there is a certain amount of admixture between the two groups. A female from one group mates with a male from her own group with a probability of $1-\mu$ and with a male from the other group with probability $\mu$. Genotypes at locus $A$ are simulated for both the parental $\left(G_{0}\right)$ and the couple $\left(G_{1}\right)$ generations, assuming no assortative mating. 1000 simulations of 200 couples in generation $G_{1}$ are performed assuming different values for $\mu$ and different sets of allelic frequency at locus $A$ in the two populations. The type-one errors of the $M$ and IBS tests when there is population stratification but no assortative mating are studied by determining the number of replicates among the 1000 where the test is significant. To compute the type-one error of the IBS test, the probabilities $P_{0}, P_{1}$ and $P_{2}$ need to be estimated. They are estimated from the data by using the genotypic frequencies in males and females in the couples analyzed, ignoring the stratification effect.

The second problem investigated is when the choice of mate is based on the geneal ogical relationship. The choice of a related mate leads to inbreeding in the population. It is different from assortative mating based on a particular locus such as HLA in that it affects all loci across the genome in the same manner. However, its effects at a given locus may be difficult to distinguish from those of positive assortative mating at that locus. Conversely, in an isolated population, avoidance of mates who are related may mimic negative assortative mating. Simulations have been performed to investigate the robustness of the two tests to inbreeding.

Power of the M test Simulations to investigate the power of the $M$ test have been performed allowing the following parameters to vary:

- the type of assortative mating: positive if spouses who share alleles preferentially mate and negative if they tend to avoid mating;

- the intensity of assortative mating: following the model developed by Hedrick ${ }^{8}$ for negative assortative mating, a female was assumed to mate preferentially with males who differ genetically from themselves with relative preferences of $1-\mathrm{s}, 1-\mathrm{hs}$ and 1 for males who are identical for 2, 1 or 0 alleles at locus $A$. The model was also used and modified for simulation of positive assortative mating;

- the number of alleles at marker $A$.
These parameters clearly have an influence: 1 . on the probability of recruiting families with informative pairs, and 2. on the power of the $M$ test to detect assortative mating.

\section{Application of the M test to data from the Hutterite population}

The $\mathrm{M}$ test has been applied to a sample of 410 couples of the Hutterite population ${ }^{5}$ for whom genotypes, as well as gen otypes of parents, were available at 16loci within the HLA region. Analysis was performed on the high resolution HLA haplotypes. ${ }^{9}$

\section{Results}

\section{Statistical properties of the M test compared with the} IBS test

Robustness of the IBS and M tests to stratification and inbreeding In Figure $1(\mathrm{a}-\mathrm{c})$, we report the type one errors of the $M$ test and of the IBS test as a function of the admixture level $\mu$ for a nominal value of $5 \%$ when there are respectively no differences in allele frequencies at the studied locus in the two populations, moderate differences and large differences. As expected, when the allelic frequencies are the same in the two populations (Figurela), type one errors for the two tests stay close to their nominal value. For moderate (Figure 1b) and large (Figure1c) differences in allelic frequencies, however, the type one error of the IBS test is increased and may even reach very high values for high admixture rates and large differences. For instance, for $\mu=0.4$, the type one error of the IBS test is respectively $8.4 \%$ and $60.2 \%$ for moderate and large differences. As expected, the type one error of the $M$ test stays very close to its nominal value in all situations clearly indicating that the test is very robust to population stratification and admixture.

As for the case of admixture, the IBS test is not robust to inbreeding, whereas the $M$ test remains very robust (data not shown).

Power of the $M$ test In Figure2, the power of the $M$ test and of the IBS test is given as a function of the selection coefficient $\mathrm{s}$ (considering $\mathrm{h}=0.5$ ) in the case of a sample of unrelated spouses. Negative assortative mating is considered in Figure $2 \mathrm{a}$ and positive assortative mating in Figure 2b. For both tests, with negative assortative mating, the power decreases when the number of alleles at the studied locus is increased. This is expected since the greater the number of alleles, the less likely spouses are to share any allele. Most of the couples are thus expected to be in the state IBS $=0$ and the increased frequency of this state due to the negative assortative mating is more difficult to detect by the IBS test. For the $M$ test, the lack of power with increasing number of alleles is mostly due to a lack of families with informative pairs (ie families where parents share an allele).

For positive assortative mating, the behavior of the tests is different. The power of the IBS test remains almost the same when the number of alleles is changed. The power of the $M$ 

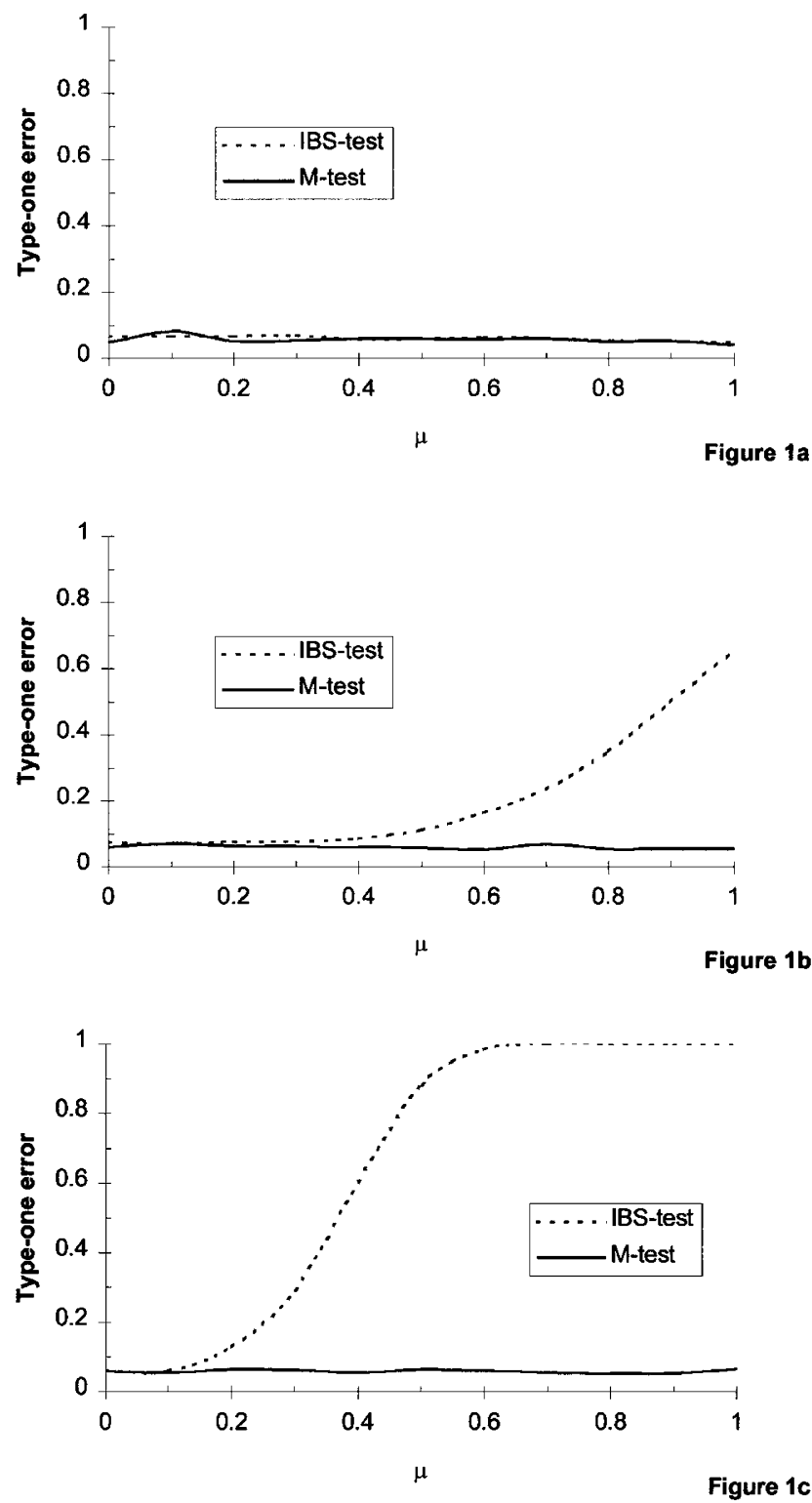

Figure 1 Type-one errors (for a nominal $P$ value of 0.05 ) of the IBS and the $M$ test as a function of the admixture level $\mu$ between the two sub-populations. 1000 simulations of 200 couples were performed in each situation. a: the two sub-populations have equal allele frequencies at the test locus ( 10 alleles, 0.1 for each allele); $\mathbf{b}$ : moderate differences, in sub-population 1 the ten alleles are equifrequent; in sub-population 2, the allelic frequencies are 0.4, 0.2, 0.05, $0.05,0.05,0.05,0.05,0.05,0.05,0.05$; c: large differences, in sub-population 1 the ten alleles are equifrequent but in sub-population 2 , only two alleles are present with frequencies 0.1 and 0.9 .

test, however, increases with increasing number of alleles. This is due mainly to the fact that when the number of alleles is increased, parents are no longer expected to share numerous alleles and thus more families are informative. For both
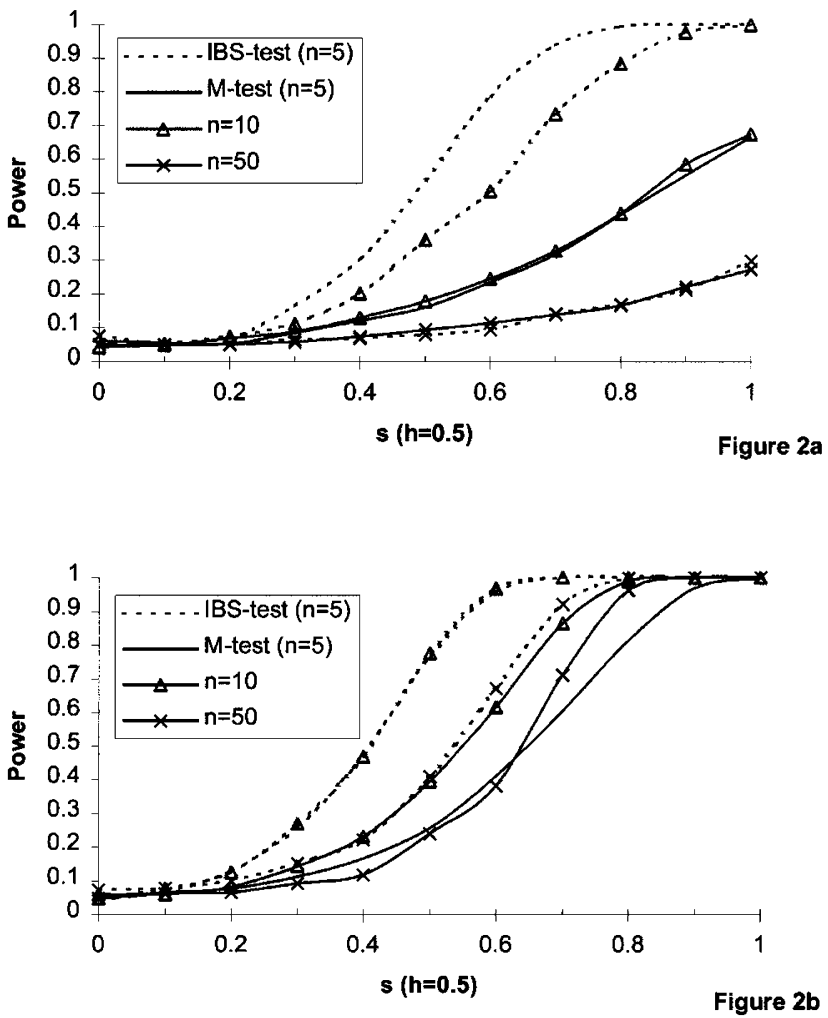

Figure 2 Power of the IBS and the $M$ test as a function of the intensity of assortative mating $\mathrm{s}$ for the case of $\mathrm{h}=0.5$. a: negative assortative mating is considered, and $\mathbf{b}$ : positive assortative mating. 1000 simulations of 200 couples were performed in each situation. Results are reported for different numbers of alleles at the locus under assortative mating ( $n=5,10$ and 50). All alleles have the same frequency. Power is given for a nominal $P$ value of 0.05 .

positive and negative assortative mating, the $M$ test appears to be less powerful than the IBS test. This is even more dramatic when the number of alleles is small.

\section{Application of the M-test to data from the Hutterite population}

Among the 410 families genotyped for the 16-locus HLA haplotypes, 79 were informative for the $M$ test ( 72 type 1 and 7 type 3 families) leading to a total of 172 informative parents. The shared allele was transmitted by 77 of these 172 parents leading to an $M$ statistic of 1.88 ( $P>0.17$ ). No difference was found based on the family type (type 1 or type3) or based on whether or not families with multiple marriages between two sibships were included (see discussion in Ober et $\mathrm{al}^{5}$ ).

We repeated the analysis stratifying on the sex of the parents in the informative pairs. In Table2, the shared allele transmission distributions are reported for the 3 different informative pairs (father-father, father-mother + motherfather (referred to as father-mother for simplicity), mothermother). Negative assortative mating was detected for both 
Table 2 Transmissions of the shared allele by informative parents in the Hutterite sample accounting for the sex of the parents in the informative pair

\begin{tabular}{|c|c|c|c|}
\hline \multirow[b]{2}{*}{ Type of informative pair } & \multicolumn{3}{|c|}{$\begin{array}{l}\text { Transmission of shared } \\
\text { allele by informative } \\
\text { parents }\end{array}$} \\
\hline & Yes & No & M test P-value \\
\hline 1 father-father & 20 & 14 & $1.06(P=0.303)$ \\
\hline mother-father & 49 & 61 & $1.31(P=0.252)$ \\
\hline father & 24 & 31 & \\
\hline mother & 25 & 30 & \\
\hline 3 mother-mother & 8 & 20 & $5.14(P=0.023)$ \\
\hline $2+3$ & 57 & 81 & $4.17(P=0.041)$ \\
\hline
\end{tabular}

The pairs are described as mother-father referring to pairs where parents carrying a shared haplotype are respectively the mother of the 1 st spouse and the father of the 2 nd spouse. The category $2+3$ combines all the informative pairs where at least one of the informative parents is a mother.

type 1 and type 3 families in mother-mother pairs $M=5.14$, $P$ value $=0.023$ ). Heterogeneity was found between fatherfather and mother-mother pairs ( $P$ value $=0.017$ ) but not between father-mother and mother-mother pairs $(P=0.125)$ and between father-father and father-mother pairs $(P=0.145)$. When grouping mother-mother and fathermother, ie pairs including at least one female informative parent, a negative assortative mating effect was still detectable ( $M=4.17, P=0.041)$. In father-mother pairs, the number of shared alleles transmitted by mothers and fathers were very similar. Moreover, no difference was found in these families between pairs where the mother was the mother of the female spouse, and pairs where the mother was the mother of the male spouse, suggesting that the avoidance of a mate carrying a maternal haplotype is not restricted to female or male only but that both sexes are choosing.

It should be noted that families where both parents of one of the spouses share a haplotype that is also shared by one of the parents of their in-law (families of the type $A_{i} A_{j}, A_{i} A_{k}$ (parents of the first spouse) and $A_{i} A_{1}, A_{m} A_{n}$ (parents of the second spouse)), have been excluded from our analyses. In these families, there may be a bias in the transmissions from parents sharing the HLA haplotype to their offspring due to maternal-fetal compatibility being deleterious in human pregnancy as shown in the Hutterites ${ }^{10}$ and this could mimic negative assortative mating. When the haplotype shared by both parents of one of the spouses is different from the hapl otype shared in the informative pair, the maternal-fetal effect should only reduce the power to detect negative assortative mating. This was the case for seven families in our sample who have not been excluded from the analyses.

Attention is drawn to the fact that in the $P$ values reported above, no account was taken of the multiple comparisons that were performed. It is indeed difficult to make any correction based on the number of tests since these tests are non-independent.

\section{Discussion}

In this paper, we have proposed a test for assortative mating, the $M$ test, which is robust to the presence of inbreeding and stratification in the population. It permits testing for assortative mating in small inbred populations as well as in admixed populations, where socio-cultural effects in mate choice may be more limited than in large panmictic populations. Although our test is robust to many different population structures, whilst the IBS test ${ }^{4,6}$ is not, it has the drawback of being less powerful than the IBS test. The $M$ test should thus be used preferentially for large samples and with highly polymorphic loci. In these situations, it competes well with other tests as illustrated here by a study in the Hutterite population.

Using the data of Ober et al, ${ }^{5-9}$ we have been able to confirm their results of negative assortative mating for HLA haplotypes and have further shown a strong sex dependence effect, which was al ready noted by Ober et al. ${ }^{5}$ The data show that in the Hutterite population, there is a preferential choice for mates who have a different HLA haplotype, as evidenced by a lack of couples sharing one haplotype in families where parents of spouses share a haplotype but only when one of the parents sharing the haplotype is female. Such a pattern could be explained by an avoidance of mates who have one haplotype similar to one of the mother's haplotypes. This would imply that individuals have the ability to recognize among potential partners those who carry an HLA haplotype similar to their own mother. The recognition could then involve a process of odor recognition as MHC genes have been shown to be involved in odor recognition in mice. $^{11,12}$

In humans, a relationship between HLA and odor recognition is not well established. Two studies by Wedekind and colleagues $^{13,14}$ suggested that preferences for body odors retained in T-shirts were associated with a degree of HLA similarity, with subjects in general preferring the odor of MHC disparate T-shirt wearers, although these studies have been criticized on methodological grounds. ${ }^{15}$ Consistent with the results of our studies in the Hutterites and Wedekind's studies ${ }^{13,14}$ are recent studies mapping human olfactory receptor genes to the telomeric end of the HLA region, ${ }^{16}$ raising the possibility that HLA-based preferences may be influenced by variation in these genes.

The effects of MHC haplotypes on mating preferences detected in the Hutterite sample are very strong. It is unlikely that this effect is due to an unusual repertoire of HLA haplotypes in the Hutterites because observed haplotypes are very similar to those found in other Caucasian populations. ${ }^{17}$ However, it is likely that the magnitude of the effect is greater in the Hutterites as a result of the relative homogeneity in this population with respect to socio-economic and cultural factors that influence mate choice in most other human populations. In addition, the limited number of haplotypes in the Hutterites increases the likelihood of encountering potential mates with haplotypes identical to one's own 
haplotype, which would be uncommon in outbred populations. Regardless of this, mate avoidance of such magnitude may have served to ensure both the avoidance of inbreeding and maintenance of variability at MHC loci in ancestral populations with sizes and structures similar to the Hutterites.

Our simulation studies have al ways assumed that assortative mating was detected at the actual locus where assortative mating was operating, ie it was implicitly assumed that the studied locus has a phenotypic expression that is recognizable by potential mates. Our test, however, would also detect an assortative mating effect at a locus in linkage disequilibrium with the tested locus. In this situation, the power to detect assortative mating will depend on the pattern of linkage disequilibrium between alleles at linked loci and will decrease as linkage disequilibrium decreases. Consequently, it could not be excluded that the results obtained in the Hutterites are due to negative assortative mating for a gene different from HLA, the alleles of which are in linkage disequilibrium with the HLA haplotypes.

\section{Acknowledgements}

This work was supported by an IN SERM post-doctoral grant (EG), NIH grant GM 35326 (EG, GT) and NIH grant HD21244 (CO). Special thanks are due to Diogo Meyer for fruitful discussions and to Mark Nelson for assistance in programming. We would like to thank two anonymous reviewers for their helpful suggestions.

\section{References}

1 Vogel F, Motulsky AG: Human Genetics: Problems and Approaches, 3rd edn. Springer-Verlag: New York, 1996.

2 Yamazaki K, Boyse EA, MikeV et al: Control of mating preferences in mices by genes in the major histocompatibility complex. J Exp Med 1976; 144: 1324-1335.
3 Rosenberg LT, Cooperman D, Payne R: HLA and mate selection. Immunogenetics 1983; 17: 89-93.

4 Jin K, Speed TP, Thomson G: Test of random mating for a highly polymorphic locus: application to HLA data. Biometrics 1995; 51: 1064-1076.

5 Ober C, Weitkamp LR, Cox N, Dytch H, Kostyu D, Elias S: HLA and mate choice in humans. Am J Hum Genet 1997; 61: 497-504.

6 Hedrick PW, Black FL: HLA and mate selection: no evidence in south Amerindians. Am J Hum Genet 1997; 61: 505-511.

7 Weitkamp LR, Ober C: Reply to Gill. Am J Hum Genet 1998; 62: 986-987.

8 Hedrick PW: Female choice and variation in the major histocompatibility complex. Genetics 1992; 132: 575-581.

9 Ober C, Weitkamp LR, Cox NJ: HLA and mate choice. In: Advances in Chemical Signals in Vertebrates. Johnston R, Muller-Schwarze D, Sorensen P (eds). Plenum Publishing Corp: New York, 1998, pp 189-199.

10 Ober C, Hyslop T, Elias S, Weitkamp LR, Hauck WW: Human leukocyte antigen matching and fetal loss: results of a 10-year prospective study. Hum Reprod 1998; 13: 33-38.

11 Boyse EA, Beauchamp GK, Bard J, Yamazaki K: Behavior and the major histocompatibility complex (MHC) H-2 of the mouse. In: Ader R, Felder DL, Cohen N (eds). Psychoneuroimmunology-II, Academic Press: San Diego, 1991, pp 831-846.

12 Beauchamp GK, Yamazaki K: HLA and mate selection in humans: commentary (invited editorial). Am J Hum Gen 1997; 61: 494-496.

13 Wedekind C, Seebeck T, Bettens F, Paepke AJ: MHC-dependent mate preferences in humans. Proc R Soc Lond B 1995; 260: 245-249.

14 Wedekind C, Füri S: Body odor preferences in men and women: do they aim for specific MHC combinations or simply heterozygosity? Proc R Soc Lond B 1997; 264: 1471-1479.

15 Hedrick P, Loeschcke V: MHC and mate selection in humans? Trends Ecol Evol 1996; 11: 24.

16 Fan W, Weiwen C, Parimoo S, Lennon GG, Weissman SM: Identification of seven new MHC classI region genes around the HLA-F locus. Immunogenetics 1996; 44: 97-103.

17 Weitkamp LR, Ober C: Ancestral and recombinant 16-locus HLA haplotypes in the Hutterites. Immunogenetics 1999; 49: 491-497. 